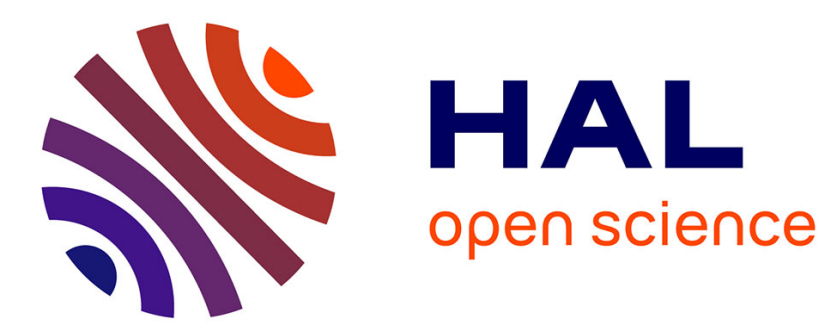

\title{
Dynamic Reliability Models for Multiple Dependent Competing Degradation Processes
}

\author{
Yan-Hui Lin, Yanfu Li, Enrico Zio
}

\section{To cite this version:}

Yan-Hui Lin, Yanfu Li, Enrico Zio. Dynamic Reliability Models for Multiple Dependent Competing Degradation Processes. ESREL 2014, Sep 2014, Wroclaw, Poland. pp.775-782. hal-01090198

\section{HAL Id: hal-01090198 \\ https://hal-centralesupelec.archives-ouvertes.fr/hal-01090198}

Submitted on 3 Dec 2014

HAL is a multi-disciplinary open access archive for the deposit and dissemination of scientific research documents, whether they are published or not. The documents may come from teaching and research institutions in France or abroad, or from public or private research centers.
L'archive ouverte pluridisciplinaire HAL, est destinée au dépôt et à la diffusion de documents scientifiques de niveau recherche, publiés ou non, émanant des établissements d'enseignement et de recherche français ou étrangers, des laboratoires publics ou privés. 


\title{
Dynamic Reliability Models for Multiple Dependent Competing Degradation Processes
}

\author{
Y.H. Lin \& Y.F. Li \\ Chair on Systems Science and the Energetic Challenge, European Foundation for New Energy - Electricité de \\ France, at École Centrale Paris - Supelec, France
}

E. Zio

Chair on Systems Science and the Energetic Challenge, European Foundation for New Energy - Electricité de France, at École Centrale Paris - Supelec, France

Energy Department, Politecnico di Milano, Milano, Italy

\begin{abstract}
This paper presents a holistic treatment to multiple dependent competing degradation processes, by employing the piecewise-deterministic Markov process (PDMP) modeling framework. The proposed method can handle the dependencies between physics-based models, between multi-state models and between these two types of models. A Monte Carlo simulation algorithm is developed to compute the components/systems reliability. A case study on one subsystem of the residual heat removal system (RHRS) of a nuclear power plant is illustrated.
\end{abstract}

\section{INTRODUCTION}

Physics-based models and multi-state models are widely used to model the degradation processes of components/systems. A physics-based model aims at developing an integrated mechanistic description of the component/system life consistent with the underlying real degradation mechanisms (e.g. wear, stress corrosion, shocks, cracking, fatigue, etc.) by using physics knowledge and equations (Chookah et al. 2011), whereas a multi-state model describes the degradation process in a discrete way, supported by material science knowledge (Li et al. 2012), degradation and/or failure data (Giorgio et al. 2011) from historical field collection or degradation tests.

Components/systems are often subject to multiple competing degradation processes and any of them may cause failure (Wang \& Pham 2012). The dependences among these processes need to be considered under certain circumstances, e.g. when the degradation state of some components/systems can influence the degradation state dynamics or lifetime distribution of other components/systems (Rasmekomen \& Parlikad 2013), or/and the various degradation processes within one component/system are physically not independent (Wang \& Pham 2012). This makes the analysis and prediction of the components/systems reliability a challenging problem (Peng et al. 2010). Wang and Pham (Wang \& Pham 2012) applied time-varying copulas for describing the dependencies between the degradation processes modeled by statistical distributions. Straub (Straub 2009) used a dynamic Bayesian network to represent the dependencies between degradation processes modeled by multi-state models. For some highly reliable components (e.g. pumps and valves in nuclear power plants, light-emitting diodes, etc.), degradation and/or failure data are often limited and do not allow building their lifetime distributions. Physics-based models are an alternative for characterizing the degradation processes (Fan et al. 2011).

In this work, we extend the existing modeling approaches to a more general setting, whereby the components degradations are modeled by physics-based models or multi-state models and dependencies exist among the components and the degradation processes within one component. To treat various dependencies, we employ the piecewise-deterministic Markov process (PDMP) (Davis 1984, Cocozza-Thivent 2011) for components and system modeling.

The remainder of this article is organized as follows. Section 2 presents the proposed degradation model for systems with degradation dependency. Monte Carlo simulation procedures to solve the model are presented in Section 3. Section 4 presents one case study on one subsystem of a residual heat removal system (Coudray \& Mattei 1984) from Électricité de France (EDF). Numerical results and analysis are presented in Section 5. Section 6 concludes the work.

\section{DEGRADATION MODELING WITH DEGRADATION DEPENDENCY}

Based on the available information/data about components degradations and failures, two main types of models are used to represent the 
degradation processes of components: physics-based model and multi-state model.

\subsection{Physics-based model}

The time-dependent evolution of continuous variables is often used to describe the degradation processes of components. The continuous variables

$\vec{X}(t)=\left(\begin{array}{l}\vec{x}(t) \\ \vec{y}(t)\end{array}\right)$

may represent: 1 . degradation variables $\vec{x}(\mathrm{t})$ such as crack length (Keedy \& Feng 2012), which describe the components degradation condition; 2. physical variables $\vec{y}(\mathrm{t})$ such as operation time, velocity and force (Daigle \& Goebel 2011a), which influence the evolution of degradation variables. Physics equations, mathematically written as differential equations (which are often a set of first-order ordinary differential equations), are then used to describe the variation of $\vec{X}(t)$ in time, considering external influencing factors $\overrightarrow{\theta_{L}}$, such as temperature and pressure (Jacobsen 2006), whose behaviors are not modeled by the physics equations.

We assume that there are $M$ components in the system, $\overrightarrow{\mathrm{L}}=\left(\mathrm{L}_{1}, \mathrm{~L}_{2}, \ldots, \mathrm{L}_{\mathrm{M}}\right)$, whose degradation processes are modeled by physics-based models. Let

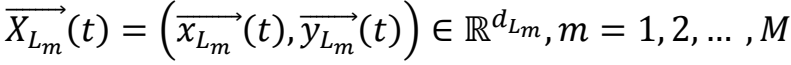

denote the vector containing all $d_{L^{m}}$ time-dependent variables for component $L_{m}$; their evolutions are described by the following first-order ordinary differential equations:

$\overrightarrow{X_{L_{m}}}(t)=\overrightarrow{f_{L_{m}}}\left(\overrightarrow{X_{L_{m}}}(t), t \mid \overrightarrow{a_{L_{m}}}\right)$

where $\overrightarrow{\mathrm{a}_{\mathrm{Lm}}}$ represents the parameters in the physics equations, including external influencing factors $\overline{\theta_{\mathrm{Lm}}}$.

To consider the dependences between the components, let

$\vec{X}(t)=\left(\overrightarrow{X_{L_{1}}}(t), \overrightarrow{X_{L_{2}}}(t), \ldots, \overrightarrow{X_{L_{M}}}(t)\right) \in$

$\mathbb{R}^{d_{L}}=\mathbb{R}^{d_{L_{1}}+d_{L_{2}}+\ldots+d_{L_{M}}}$

denote the overall degradation condition of all the $M$ components. We extend the set of differential equations in (3) by including the variables of the other $M-1$ components:

$\overrightarrow{X_{L_{m}}}(t)=\overrightarrow{\overrightarrow{f_{L_{m}}}}\left(\vec{X}(t), t \mid \overrightarrow{a_{L_{m}}}, \overrightarrow{b_{L_{m}}}\right)$

where $\overline{\mathrm{b}_{L m}}$ is the vector of parameters characterizing the influence of the other components on the degradation processes of component $L_{m}$.

For any component $L_{m}$, the degradation variables

$\overrightarrow{x_{L_{m}}}(t)=\left(x_{L_{m}}^{1}(t), x_{L_{m}}^{2}(t), \ldots, x_{L_{m}}^{d_{L_{m}}^{1}}(t)\right)$

have the critical values (thresholds):

${\overrightarrow{L_{L_{m}}}}^{*}=\left(x_{L_{m}}^{1}{ }^{*}, x_{L_{m}}^{2}{ }^{*}, \ldots, x_{L_{m}}^{d_{L_{m}}^{1}{ }^{*}}\right)$

Component $L_{m}$ fails when one of the degradation variables exceeds the corresponding critical value. The failure time $t_{f}$ of component $L_{m}$ is defined as follows:
$t_{f}=\operatorname{Min}_{\arg t}\left[x_{L_{m}}^{1}(t)=x_{L_{m}}^{1}{ }^{*}, \ldots, x_{L_{m}}^{d_{L_{m}}^{1}}(t)=x_{L_{m}}^{d_{L_{m}}^{1}{ }^{*}}\right]$

\subsection{Multi-state model}

Under the situation that there is not any available physics-based model for the degradation processes of the components, a multi-state model (Lisnianski \& Levitin 2003) can be built using historical degradation and/or failure data of components. Markov processes (Giorgio et al. 2011) and semi-Markov processes (Chryssaphinou et al. 2011) are widely used in practice as multi-state models, where the degradation conditions of the components are represented by a limited number of states describing the degradation severity. The transition rates (estimated from historical data) between two different degradation states are used to describe the speed of reaching another degradation state.

We assume that there are $N$ components in the system, $\overrightarrow{\mathrm{K}}=\left(\mathrm{K}_{1}, \mathrm{~K}_{2}, \ldots, \mathrm{K}_{\mathrm{N}}\right)$, whose degradation processes are modeled by multi-state models. The component $K_{n}$ has $d_{k n}+1$ states, $\mathrm{S}_{\mathrm{Kn}}=\left\{0,1, \ldots, \mathrm{d}_{\mathrm{Kn}}\right\}$, ranging from perfect functioning state ' $d_{k n}$ ' to complete failure state ' 0 '. The component is partially functioning in all generic intermediate states.

To consider the dependences between the components and the influencing factors, let

$\vec{Y}(t)=\left(Y_{K_{1}}(t), Y_{K_{2}}(t), \ldots, Y_{K_{N}}(t)\right) \in$

$S=S_{K_{1}} \times S_{K_{2}} \ldots \times S_{K_{N}}$

denote the overall degradation condition of all the $N$ components, where $\mathrm{Y}_{\mathrm{Kn}}(\mathrm{t}), \mathrm{n}=1,2, \ldots, \mathrm{N}$, indicates the degradation state of component $K_{n}$ at time $t$. Let $\left\{\overrightarrow{\mathrm{Y}_{\mathrm{k}}}, \mathrm{T}_{\mathrm{k}}\right\} \in \mathrm{S} \times \mathbb{R}^{+}, \mathrm{k} \in \mathbb{N}$ denote the degradation processes of the $N$ components, where $\overrightarrow{\mathrm{Y}_{\mathrm{k}}}$ denotes the state of the $N$ components after $k$ transitions (a transition occurs as long as any one of the $N$ components changes its state) and $T_{k}$ denotes the time of arrival at state $\overrightarrow{\mathrm{Y}_{\mathrm{k}}}$. Then, the state of the $N$ components at time $t$ can be defined as follows:

$\vec{Y}(t)=\overrightarrow{Y_{k}}, \forall t \in\left[T_{k}, T_{k+1}[\right.$

We assume that $\left\{\overrightarrow{\mathrm{Y}}_{\mathrm{k}}, \mathrm{T}_{\mathrm{k}}\right\}, \mathrm{k} \geq 0$ follows a Markov renewal process which generalizes the notion of Markov jump process. Then, the probability that the $\mathrm{N}$ components will step to state $\overrightarrow{\mathrm{j}}$ from state $\overrightarrow{\mathrm{l}}$ in the time interval $\left[\mathrm{T}_{\mathrm{n}}, \mathrm{T}_{\mathrm{n}}+\Delta \mathrm{t}\right]$, given $\left\{\overrightarrow{\mathrm{Y}_{\mathrm{k}}}, \mathrm{T}_{\mathrm{k}}\right\}, \mathrm{k} \leq \mathrm{n}$, is defined as follows:

$$
\begin{aligned}
& P\left[\overrightarrow{Y_{n+1}}=\vec{\jmath}, T_{n+1} \in\left[T_{n}, T_{n}+\Delta t\right] \mid\left\{\overrightarrow{Y_{k}}, T_{k}\right\}_{k \leq n}, \overrightarrow{\theta_{K}}\right] \\
& =P\left[\overrightarrow{Y_{n+1}}=\vec{\jmath}, T_{n+1} \in\left[T_{n}, T_{n}+\Delta t\right] \mid \overrightarrow{Y_{n}}=\vec{\imath}, \overrightarrow{\theta_{K}}\right] \\
& \forall n \geq 0, \overrightarrow{\Delta t} \geq 0, \vec{\imath}, \vec{\jmath} \in S, \vec{\imath} \neq \vec{\jmath}
\end{aligned}
$$

where $\overrightarrow{\theta_{\mathrm{K}}}$ represents the external influencing factors. The process $\vec{Y}(t)$ is a continuous time Markov process if the holding time at any state $\vec{\imath} \in S$ is exponentially distributed according to state $\vec{\imath}$ and the system total running time $t$, denoting by the transition rate $\lambda_{\vec{i}}\left(t, \overrightarrow{\theta_{K}}\right)$. Otherwise, it is a 
semi-Markov process. The system fails if one of the $N$ components reaches its complete failure state. The reliability of such system at time $t$ can be calculated as follows:

$R(t)=P\left[Y_{K_{1}}(s) \neq d_{K_{1}}, Y_{K_{2}}(s) \neq d_{K_{2}}, \ldots, Y_{K_{N}}(s) \neq\right.$

$\left.d_{K_{N}} \forall s \leq t\right]$

\subsection{PDMP for systems with degradation dependency}

In this section, we consider that the system contains $M$ components described by physics-based models and $N$ components described by multi-state models. The interdependencies between the two groups of components are taken into account. We assume that the degradation levels of the components in the first group may influence the transition times and transition directions of the degradation processes of the second group, and the degradation states of the second group may influence the evolution trajectories of the continuous variables in the first group. We employ PDMP to model this type of interdependence. Let

$\vec{Z}(t)=\left(\begin{array}{c}\overrightarrow{X_{L_{1}}}(t) \\ \overrightarrow{X_{L_{2}}}(t) \\ \vdots \\ \overrightarrow{X_{L_{M}}}(t) \\ \vec{Y}(t)\end{array}\right)=\left(\begin{array}{c}\vec{X}(t) \\ \vec{Y}(t)\end{array}\right) \in E=\mathbb{R}^{d_{L}} \times S$

represent the degradation condition of the whole system defined on $E$, a hybrid space of $\mathbb{R}^{d_{L}}$ and $S$. $\left(\overrightarrow{Z_{t}}=\vec{Z}(t)\right) t \geq 0$ is a stochastic process with values in the probability space $(E, \varepsilon)$ where $\varepsilon$ is a $\sigma$-algebra of $E$ (Cocozza-Thivent 2011). Let

$\overrightarrow{Z_{k}}=\vec{Z}\left(T_{k}\right)=\left(\begin{array}{c}\vec{X}\left(T_{k}\right) \\ \vec{Y}_{k}\end{array}\right) \in E, k \in \mathbb{N}$

denote the degradation state of the whole system at the transition time $T_{k}$ of the process $\vec{Y}(t)$. $\left\{\overrightarrow{\mathrm{Z}_{\mathrm{k}}}, \mathrm{T}_{\mathrm{k}}\right\}, \mathrm{k} \geq 0$ is then a Markov renewal process defined on the space $E \times \mathbb{R}^{+}$, since the probability that the whole system will step to state $\overrightarrow{\mathbf{J}^{\prime}}$ from state $\overrightarrow{\mathrm{l}^{\prime}}$ in the time interval $\left[\mathrm{T}_{\mathrm{n}}, \mathrm{T}_{\mathrm{n}}+\Delta \mathrm{t}\right]$, given $\left\{\overrightarrow{\mathrm{Z}_{\mathrm{k}}}, \mathrm{T}_{\mathrm{k}}\right\} \mathrm{k} \leq \mathrm{n}$ is as follows:

$P\left[\overrightarrow{Z_{n+1}}=\overrightarrow{J^{\prime}}, T_{n+1} \in\left[T_{n}, T_{n}+\Delta t\right] \mid\left\{\overrightarrow{Z_{k}}, T_{k}\right\}_{k \leq n}, \overrightarrow{\theta_{K}}\right]$

$=P\left[\overrightarrow{Z_{n+1}}=\overrightarrow{\jmath^{\prime}}, T_{n+1} \in\left[T_{n}, T_{n}+\Delta t\right] \mid \overrightarrow{Z_{n}}=\overrightarrow{\iota^{\prime}}, \overrightarrow{\theta_{K}}\right]$

$\forall n \geq 0, \overrightarrow{\imath^{\prime}}, \overrightarrow{\jmath^{\prime}} \in E, \overrightarrow{\imath^{\prime}} \neq \overrightarrow{\jmath^{\prime}}$

where the transition probability depends also on the variables of the $M$ components from the first group. The evolution of the whole system between two consequent jumps of process $\overrightarrow{\mathrm{Z}}(\mathrm{t})$, when $t$ $\in\left[\mathrm{T}_{\mathrm{k}}, \mathrm{T}_{\mathrm{k}+1}[, \mathrm{k} \in \mathbb{N}\right.$ is defined as follows:

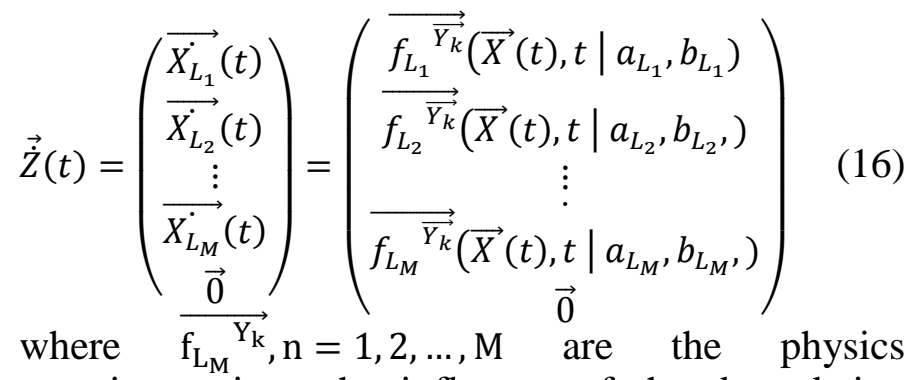
equations, given the influence of the degradation state $\overrightarrow{\mathrm{Y}_{\mathrm{k}}}$ of the $N$ components from the second group. The process $\vec{Z}(t)$ that takes values in $E$ is a Piecewise-Deterministic Process (PDP) since it can be written as follows [Lorton et al. 2013]:

$\vec{Z}(t)=\varphi\left(\overrightarrow{Z_{k}}, t-T_{k}\right)$,

for $t \in\left[T_{k}, T_{k+1}[, \forall k \in \mathbb{N}\right.$

and $\overrightarrow{\mathrm{Z}}(\mathrm{t})$ is a PDMP on the condition that $\varphi$ satisfies the following [Cocozza-Thivent. 2011]:

$\varphi(\vec{y}, t+s)=\varphi(\varphi(\vec{y}, t), s), \forall t, s \geq 0, \vec{y} \in E$

This is especially true in our case, as $\varphi$ is the solution of a first-order ordinary differential equations system [Lorton et al. 2013].

Let $\mathcal{F}=\mathcal{F}_{\overrightarrow{\mathrm{X}}} \times \mathcal{F}_{\overrightarrow{\mathrm{Y}}} \subsetneq \mathrm{E}$ denote the space of the failure states of $\vec{Z}(\mathrm{t})$, where $\mathcal{F}_{\overrightarrow{\mathrm{X}}}$ denotes the sub-space of the failure states for $\vec{X}(t)$ and $\mathcal{F}_{\overrightarrow{\mathrm{Y}}}$ denote the sub-space of the failure states for $\vec{Y}(t)$; then, the reliability of the system at time $t$ can be calculated as follows:

$R(t)=P[\vec{Z}(s) \notin \mathcal{F}, \forall s \leq t]$

\section{RELIABILITY ESTIMATION}

Analytically solving the PDMP is a difficult task due to the complex behavior of the system (Marseguerra \& Zio 1996), which contains the stochasticities in the components modeled by multi-state models and the time-dependent evolutions of the components modeled by physics-based models. On the other hand, Monte Carlo simulation methods are suited for the reliability estimation of the system.

\subsection{The simulation procedure}

The procedure of the MC simulation is represented as follows:

Set $N_{\max }$ (the maximum number of replications) and $k=0$ (index of replication)

Set $k^{\prime}=0$ (number of trials that end in the failure state)

While $k<N_{\max }$

Initialize the system by setting $\overrightarrow{Z^{\prime}}$ (initial state), and the time $T=0$ (initial system time)

Set $t^{\prime}=0$ (state holding time)

While $T<T_{\text {miss }}$

Sample a $t^{\prime}$ through the probability density function 
Sample an arrival state $\overrightarrow{Y^{\prime}}$ for stochastic process $\vec{Y}(t)$ from all the possible states through the conditional probability function

Set $T=T+t^{\prime}$

Calculate $\vec{X}(T)$ by using the physics equations (16)

Set

If $T \leq T_{\text {miss }}$

$$
\overrightarrow{Z^{\prime}}=\left(\begin{array}{c}
\vec{X}(T) \\
\overrightarrow{Y^{\prime}}
\end{array}\right)
$$

Calculate all the extreme values $\left(\vec{X}_{n}^{o}, n \in \mathbb{N}\right)$ of $\vec{X}(t)$ in the interval $\left[T-t^{\prime}, T\right]$ by using the physics equations (16)

$$
\begin{aligned}
& \text { If }\left(\exists m \in \mathbb{N}, \vec{X}_{m}^{o} \in \mathcal{F}_{X}\right) \vee\left(\overrightarrow{Z^{\prime}} \in \mathcal{F}\right) \\
& \text { Set } k^{\prime}=k^{\prime}+1 \\
& \text { Break }
\end{aligned}
$$

End if

Else (when $T>T_{\text {miss }}$ )

Calculate all the extreme values $\left(\vec{X}_{n}^{o}, n \in \mathbb{N}\right)$ of $\vec{X}(t)$ in the interval $\left[T-t^{\prime}, T_{m i s s}\right]$ by using the physics equations (16)

\section{End if \\ End While \\ Set $k=k+1$ \\ End While $\square$}

If $\exists m \in \mathbb{N}, \vec{X}_{m}^{o} \in \mathcal{F}_{X}$

Set $k^{\prime}=k^{\prime}+1$

End if

\section{Break}

Since the first derivative of $\overrightarrow{\mathrm{X}}(\mathrm{t})$ on the interval $\left[\mathrm{T}_{\mathrm{k}}, \mathrm{T}_{\mathrm{k}+1}\right], \mathrm{k} \in \mathbb{N}$ exists, $\overrightarrow{\mathrm{X}}(\mathrm{t})$ is continuous on such interval. If $\overrightarrow{\mathrm{X}}(\mathrm{t})$ is monotonous in the interval $\left[\mathrm{T}_{\mathrm{k}}, \mathrm{T}_{\mathrm{k}+1}\right]$, the extreme values of $\overrightarrow{\mathrm{X}}(\mathrm{t})$ are reached at the boundary points of the interval. Otherwise, the extreme values of $\vec{X}(t)$ can be obtained by comparing all the local extreme values found by Fermat theorem (Bronshtein et al. 1985). To calculate the value of $\vec{X}(t)$, Runge-Kutta methods can be applied for the numerical solution of the ordinary differential equations (Hairer et al. 1993, Hairer et al. 1996).

The estimated component reliability at time $\mathrm{T}_{\text {miss }}$ can be obtained by

$\hat{R}\left(T_{\text {miss }}\right)=1-k^{\prime} / N_{\text {max }}$

where $k^{\prime}$ represents the number of trials that end in the failure state of the system, and the sample variance is (Lewis \& Böhm 1984):

$\operatorname{var}_{\hat{R}\left(T_{\text {miss }}\right)}=\hat{R}\left(T_{\text {miss }}\right)\left(1-\hat{R}\left(T_{\text {miss }}\right)\right) /\left(N_{\text {max }}-1\right)$

\section{ILLUSTRATIVE CASE}

In this section, one important subsystem of a residual heat removal system (Coudray \& Mattei 1984) has been considered to illustrate the proposed method. This subsystem consists of a pneumatic valve and a centrifugal pump, which are used in conjunction in a variety of domains for fluid delivery. The degradation model of the pump is modified from the one originally supplied by EDF, while that of the valve is an existent physics-based model presented in (Daigle \& Goebel 2010, Lorton et al. 2013). Two types of dependence have been considered, as follows:

1. Common degradation cause: the abrasive particles in the fluid which can aggravate the degradation processes of both components (Wierman et al. 2007).

2. Degradation dependency: the degradation of the pump can lead it to vibrate (Zhang et al. 2006), which will, in turn, cause the vibration of the valve and therefore aggravate the degradation process of the latter (Moussou et al. 2001).

The common degradation cause and degradation dependency leads us to resort to our proposed method to assess the reliability of this two-component series system.

\subsection{Pneumatic valve}

The pneumatic valve refers to a normally-closed and gas-actuated valve with a linear cylinder actuator which has been studied by Daigle and Goebel (Daigle \& Goebel 2010) and Lorton et al. (Lorton et al. 2013) by using physics-based modeling. A simplified scheme of the valve is shown in Figure 1.

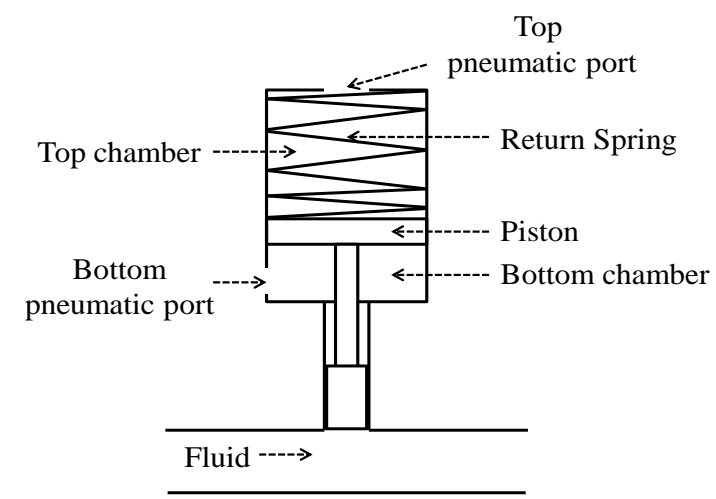

Figure 1. Simplified scheme of the pneumatic valve (Daigle \& Goebel 2010).

The valve contains a top chamber (above the piston) and a bottom chamber (below the piston), which are respectively connected to a top pneumatic port and a bottom pneumatic port. The position of the piston (between fully closed position ' 0 ' and fully open position ' $L_{s}$ ') is controlled by filling or evacuating the chambers by regulating the pressure of the pneumatic ports. A return spring is linked with the piston to ensure the closure of the valve when pressure is lost due to the spring force.

One of the most common degradation mechanisms of the valve is the internal leak at the seal surrounding the piston (Lorton et al. 2013). 
Owing to the internal leak of the piston, the pneumatic gas can flow between the two chambers therefore influencing the response time and the behavior of the valve. The degradation variable of the valve is then the equivalent orifice area of the internal leak of the piston, denoted by $\mathrm{L}(\mathrm{t})$. At the initial stage, the valve is set to the fully closed position.

The degradation process of the valve at time $t$ is described by the following vector:

$\overrightarrow{X_{v}}(t)=\left(\begin{array}{c}L(t) \\ x(t) \\ v(t) \\ m_{t}(t) \\ m_{b}(t) \\ t\end{array}\right)$

where $x(t)$ is the position of the valve, $v(t)$ is the velocity of the valve, $m_{t}(t)$ is the mass of the gas in the top chamber, $m_{b}(t)$ is the mass of the gas in the bottom chamber and $t$ is the running time of the valve. The derivatives of these variables are represented by:

$\overrightarrow{\dot{X}_{v}}(t)=\left(\begin{array}{c}\dot{L}(t) \\ v(t) \\ a(t) \\ f_{t}(t) \\ f_{b}(t) \\ 1\end{array}\right)$

where $a(t)$ is the valve acceleration, $f_{t}(t)$ and $f_{b}(t)$ are the mass flows going into the top and bottom chambers, respectively. The development of the internal leak is defined as follows:

$\dot{L}(t)=w\left(1+\alpha_{v}\right) r v(t)^{2}$

where $v(t)$ is the velocity of the valve, $\mathrm{w}$ is the wear coefficient without the consideration of abrasive particles in the fluid, and $\alpha_{\mathrm{v}}$ is a constant to characterize the relative increment of the developing rate of the internal leak influenced by the abrasive particles in the fluid. All the other physics equations of the valve and the parameter definitions and values can be founded in (Daigle \& Goebel 2010).

The critical value $L^{*}$ for the internal leak of the piston $\mathrm{L}(\mathrm{t})$ is defined as the value above which $\left(\mathrm{L}(0)>\mathrm{L}^{*}\right)$ the valve cannot reach the fully open position within the $15 \mathrm{~s}$ time limit after an opening command is executed at time $t=0 \mathrm{~s}$. The size of the internal leak is assumed to be constant during the opening procedure $(\dot{L}(\mathrm{t})=0,0 \leq \mathrm{t} \leq 15)$ (Lorton et al. 2013) to obtain a conservative critical value that $\mathrm{L}^{*}=3.20 \mathrm{e}-6 \mathrm{~m}^{2}$ in this case. The behavior of the valve within 15 s with different values of $L(0)$ is shown in Figure 2.

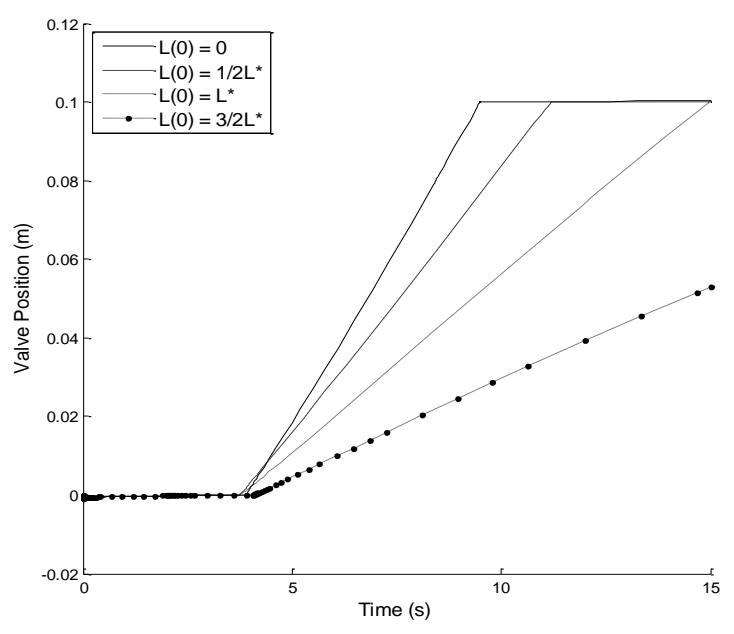

Figure 2. Valve behavior with different sizes of the internal leak.

\subsection{Centrifugal pump}

We assume that the degradation process of the centrifugal pump is modeled by a continuous-time homogeneous Markov chain with constant transition rates as shown in Figure 3:

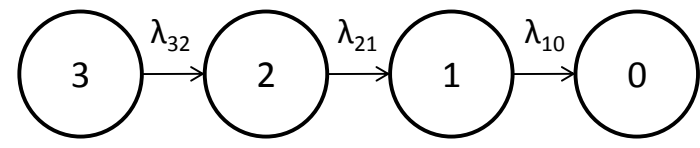

Figure 3. Degradation process of the pump.

The perfect functioning state is denoted with the label ' 3 ' and ' 0 ' is the label of the complete failure state. At the initial stage, the valve is set to the perfect functioning state. The vibration of the pump caused by degradation is classified into two levels: 'smooth' and 'rough' (Vlok et al. 2002) corresponding to the degradation states ' 2 ' and ' 1 ', respectively. Let $Y_{p}(t)$ denote the degradation state of the pump at time $t$ and $S_{p}=\{0,1,2,3\}$ denote the degradation states set. The formulations of transition rates are assumed as follows:

$\left\{\begin{array}{l}\lambda_{32}=\lambda_{32}^{\prime}\left(1+\alpha_{p 32}\right) \\ \lambda_{21}=\lambda_{21}^{\prime}\left(1+\alpha_{p 21}\right) \\ \lambda_{10}=\lambda_{10}^{\prime}\left(1+\alpha_{p 10}\right)\end{array}\right.$

where $\lambda_{32}^{\prime}, \lambda_{21}^{\prime}$ and $\lambda_{10}^{\prime}$ are the original transition rates without the consideration of abrasive particles in the fluid. The constants $\alpha_{\mathrm{p} 32}, \alpha_{\mathrm{p} 21}$ and $\alpha_{\mathrm{p} 10}$ represent the relative increments of transition rates caused by the abrasive particles in the fluid. 


\subsection{PDMP for the degradation processes of the system}

The degradation processes of the whole system are modeled by PDMP as follows:

$\vec{Z}(t)=\left(\begin{array}{l}\overrightarrow{X_{v}}(t) \\ Y_{p}(t)\end{array}\right)=\left(\begin{array}{c}L(t) \\ x(t) \\ v(t) \\ m_{t}(t) \\ m_{b}(t) \\ t \\ Y_{p}(t)\end{array}\right) \in R^{6} \times S_{p}$

and

$$
\overrightarrow{\dot{Z}}(t)=\left(\begin{array}{c}
\overrightarrow{X_{v}}(t) \\
0
\end{array}\right)=\left(\begin{array}{c}
\dot{L}^{\prime}\left(t, Y_{p}(t)\right) \\
v(t) \\
a(t) \\
f_{t}(t) \\
f_{b}(t) \\
1 \\
0
\end{array}\right)
$$

where $\dot{L}^{\prime}\left(t, Y_{p}(t)\right)$ is the derivative of the internal leak of the valve with the consideration of the degradation dependency between the valve and the pump while the development of the internal leak of the valve is dependent on the degradation state of the pump, we have

$\dot{L}^{\prime}\left(t, Y_{p}(t)\right)=w\left(1+\alpha_{v}\right)\left(1+\beta_{Y_{p}(t)}\right) r v(t)^{2}$

where $\beta_{Y \mathrm{p}(t)}$ is the relative increment of the developing rate of the internal leak caused by the vibration of the pump (if we ignore the degradation dependency, then $\left.\beta_{Y p(t)}=0\right)$. The times between two consequent jumps of PDMP follow the exponential distribution with constant transition rates determined by equation (25). The space of the failure states of $\vec{Z}(t)$ is $\mathcal{F}=\mathcal{F}_{\overrightarrow{X v}} \times \mathcal{F}_{Y p}=\left[L^{*},+\infty\right) \times\{0\}$.

\section{RESULTS AND ANALYSIS}

Due to the high reliability of the components, it is typically computationally expensive to simulate the failures or certain degradations. The values of the parameters of the pump and of the parameters characterizing the degradation dependency are shown in Table 1 . We set them in a way to simulate the system functioning under an accelerated aging condition.

Table 1. The values of the parameters of the pump and of the parameters characterizing the degradation dependency.

\begin{tabular}{ll}
\hline Parameter & Value \\
\hline$w$ & $5 \mathrm{e}-9 \mathrm{~m} / \mathrm{N}$ \\
$\alpha_{v}$ & $20 \%$ \\
$\lambda_{32}^{\prime}$ & $5.00 \mathrm{e}-3 / \mathrm{s}$ \\
$\lambda_{21}^{\prime}$ & $5.00 \mathrm{e}-3 / \mathrm{s}$ \\
$\lambda_{10}^{\prime}$ & $5.00 \mathrm{e}-3 / \mathrm{s}$
\end{tabular}

\begin{tabular}{ll}
$\alpha_{p 32}$ & $20 \%$ \\
$\alpha_{p 21}$ & $20 \%$ \\
$\alpha_{p 10}$ & $20 \%$ \\
$\beta_{3}$ & 0 \\
$\beta_{2}$ & $10 \%$ \\
$\beta_{1}$ & $20 \%$ \\
$\beta_{0}$ & 0 \\
\hline
\end{tabular}

At the initial stage, the two components are both in perfect state and the valve is in the fully closed position. The command of the valve is a 30s-periodic-signal and the valve is commanded to open in the first half-period and to close in the second half. The pump is functioning until it reaches the failure state ' 0 '.

A Monte Carlo simulation over a time horizon of $\mathrm{T}_{\text {miss }}=700 \mathrm{~s}$ is run $\mathrm{N}_{\max }=10^{5}$ times. In Figure 4, we compare the reliability of the whole system with common degradation cause and degradation dependency to that with only common degradation cause. From the Figure, we can see that before $465.67 \mathrm{~s}$ (point A) the two curves coincide and the system reliability is equal to the reliability of the pump. After that time, the valve begins to fail in some simulation trials, which corresponds to the situation when the pump jumps to state ' 1 ' very quickly and stays there until the valve fails. The system reliability, then, experiences three sharp decreases at around 497.39s (point B), 526.77s (point $\mathrm{C}$ ) and $556.45 \mathrm{~s}$ (point D) respectively, and the system is definitely failed afterwards. The longest failure time of the valve is at point $\mathrm{D}$, corresponding to the situation when the pump stays in the initial state ' 3 ' from the beginning until the failure of the valve.

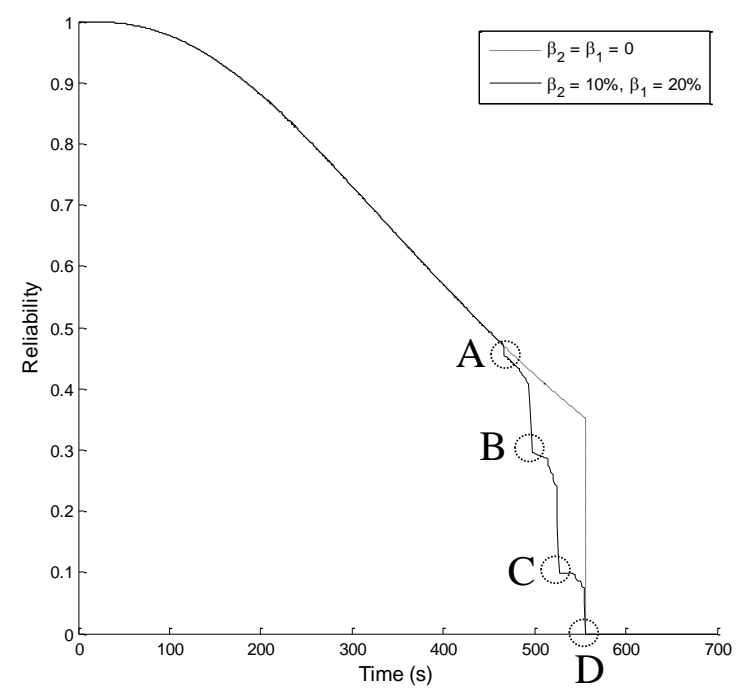

Figure 4. System reliability with common degradation cause and w/o degradation dependency.

The development of the internal leak of the valve during one simulation trial is presented in Figure 5, 
where the pump is seen to reach state ' 2 ' at $152.83 \mathrm{~s}$, state ' 1 ' at $439.92 \mathrm{~s}$ and failure state ' 0 ' at $635.78 \mathrm{~s}$. The valve fails at $525.03 \mathrm{~s}$ which is $31.28 \mathrm{~s}$ earlier than the failure time without degradation dependency.
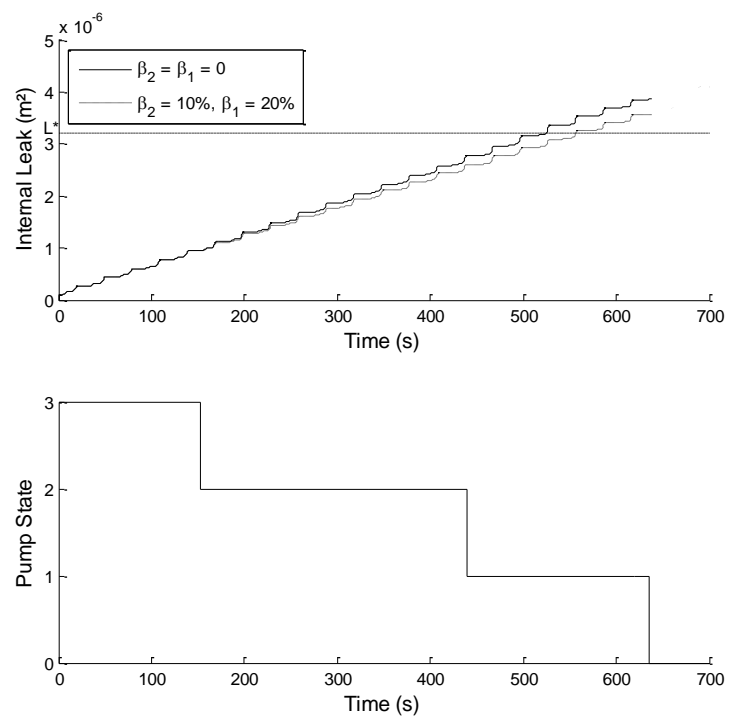

Figure 5. Development of the internal leak of the valve in one simulation trial with common degradation cause and w/o degradation dependency.

\section{CONCLUSION}

We employ the PDMP approach to develop a new method for modeling multiple dependent competing degradation processes. The significance of the proposed method lies in its capability to describe the degradation dependency between physics-based models, between multi-state models and between the two types of models. A Monte Carlo simulation algorithm is designed to compute the components/systems reliability. The results of a illustrative case study have shown the effectiveness and capabilities of our modeling and simulation framework.

\section{ACKNOWLEDGEMENT}

The authors would like to thank Mrs. Dominique VASSEUR, Mr. Antoine DESPUJOLS and $\mathrm{Mr}$. Emmanuel ARDILLON from the Department Industrial Risks Management (MRI), Electricité de France R\&D for their insightful comments. The work was supported by Electricité de France Contract No.8610-5910112096.

\section{REFERENCES}

Bronshtein, I. N., Semendiaev, K. A. \& Hirsch, K. A. 1985. Handbook of mathematics, Van Nostrand Reinhold New York.

Chookah, M., Nuhi, M. \& Modarres, M. 2011. A probabilistic physics-of-failure model for prognostic health management of structures subject to pitting and corrosion-fatigue. Reliability Engineering \& System Safety, 96, 1601-1610.

Chryssaphinou, O., Limnios, N. \& Malefaki, S. 2011. Multi-state reliability systems under discrete time semi-Markovian hypothesis. Reliability, IEEE Transactions on, 60, 80-87.

Cocozza-Thivent, C. 2011. Processus de renouvellement markovien, Processus de Markov déterministes par morceaux. Online book available on the webpage: http://perso-math. univ-m/v. fr/users/cocozza. christiane/recherche-pageperso/PresentationRMetPD MP. html.

Coudray, R. \& Mattei, J. 1984. System reliability: An example of nuclear reactor system analysis. Reliability Engineering, 7, 89-121.

Daigle, M. \& Goebel, K. Model-based prognostics under limited sensing. Aerospace Conference, 2010 IEEE, 2010. IEEE, 1-12.

Daigle, M. \& Goebel, K. 2011a. A model-based prognostics approach applied to pneumatic valves. International Journal of Prognostics and Health Management, 2, 1-16.

Davis, M. H. 1984. Piecewise-deterministic Markov processes: A general class of non-diffusion stochastic models. Journal of the Royal Statistical Society. Series $B$ (Methodological), 353-388.

Fan, J., Yung, K. \& Pecht, M. 2011. Physics-of-failure-based prognostics and health management for high-power white light-emitting diode lighting. Device and Materials Reliability, IEEE Transactions on, 11, 407-416.

Giorgio, M., Guida, M. \& Pulcini, G. 2011. An age-and state-dependent Markov model for degradation processes. IIE Transactions, 43, 621-632.

Hairer, E., Nørsett, S. P. \& Wanner, G. 1993. Solving Ordinary Differential Equations I: Nonstiff Problems, Springer.

Hairer, E., Nørsett, S. P. \& Wanner, G. 1996. Solving Ordinary Differential Equations II: Stiff and Differential-Algebraic Problems, Springer.

Jacobsen, M. 2006. Point process theory and applications: marked point and piecewise deterministic processes, Springer.

Keedy, E. \& Feng, Q. 2012. A physics-of-failure based reliability and maintenance modeling framework for stent deployment and operation. Reliability Engineering \& System Safety, 103, 94-101.

Lewis, E. \& Böhm, F. 1984. Monte Carlo simulation of Markov unreliability models. Nuclear Engineering and Design, 77, 49-62.

Li, Y.-F., Zio, E. \& Lin, Y.-H. 2012. A Multistate Physics Model of Component Degradation Based on Stochastic Petri 
Nets and Simulation. Reliability, IEEE Transactions on, 61, $921-931$.

Lisnianski, A. \& Levitin, G. 2003. Multi-state system reliability: assessment, optimization and applications, World scientific.

Lorton, A., Fouladirad, M. \& Grall, A. 2013. Computation of remaining useful life on a physic-based model and impact of a prognosis on the maintenance process. Proceedings of the Institution of Mechanical Engineers, Part O: Journal of Risk and Reliability.

Marseguerra, M. \& Zio, E. 1996. Monte Carlo approach to PSA for dynamic process systems. Reliability Engineering \& System Safety, 52, 227-241.

Moussou, P., Cambier, S., Lachene, D., Longarini, S., Paulhiac, L. \& Villouvier, V. 2001. Vibration investigation of a French PWR power plant piping system caused by cavitating butterfly valves. ASME-PUBLICATIONS-PVP, 420, 99-106.

Peng, H., Feng, Q. \& Coit, D. W. 2010. Reliability and maintenance modeling for systems subject to multiple dependent competing failure processes. IIE transactions, 43, 12-22.

Rasmekomen, N. \& Parlikad, A. K. 2013. Maintenance Optimization for Asset Systems With Dependent Performance Degradation. Reliability, IEEE Transactions on, 62, 362 - 367.

Straub, D. 2009. Stochastic modeling of deterioration processes through dynamic Bayesian networks. Journal of Engineering Mechanics, 135, 1089-1099.

Vlok, P., Coetzee, J., Banjevic, D., Jardine, A. \& Makis, V. 2002. Optimal component replacement decisions using vibration monitoring and the proportional-hazards model. Journal of the operational research society, 193-202.

Wang, Y. \& Pham, H. 2012. Modeling the dependent competing risks with multiple degradation processes and random shock using time-varying copulas. Reliability, IEEE Transactions on, 61, 13-22.

Wierman, T., Rasmuson, D. M. \& Mosleh, A. 2007. Common-cause failure database and analysis system: event data collection, classification, and coding, Division of Risk Assessment and Special Projects, Office of Nuclear Regulatory Research, US Nuclear Regulatory Commission.

Zhang, S., Hodkiewicz, M., Ma, L. \& Mathew, J. 2006. Machinery condition prognosis using multivariate analysis. Engineering Asset Management. Springer. 